\title{
Numerical Analysis of the New Concept of Screw of an Extruder for Polymer Materials
}

\author{
H. Debski ${ }^{1, *}$, P. Wysmulski ${ }^{1}$ \\ 'Department of Machine Design and Mechatronics, Lublin University of Technology, Nadbystrzycka str.36, Lublin, Poland
}

\begin{abstract}
The article introduces an innovative concept of a screw of an extruder used for granulation of polymer materials. Three construction solutions were introduced, differing in the construction parameters of the screw. The scope of the research included the numerical strength-thermal analysis of the introduced construction solutions of the screw. The analysis was conducted using the finite element method. Numerical calculations were a thermally coupled FEM analysis, posing a geometrically and thermally nonlinear problem. The obtained results confirmed the sufficient strength of the structure of the screw, subjected to both the pressure caused by the transport of polymer granules as well as the temperature generated by the friction of the granules on the surface of the screw. The numerical tool applied in the research was the commercial program ABAQUS ${ }^{\text {. }}$
\end{abstract}

Keywords: polymer materials, granules, screw of the extrusion press, finite element method.

\section{Introduction}

An important aspect of developing modern extrusion presses for production of elements made of polymer materials is a proper choice of the geometrical parameters of the press, which allows one to obtain the optimal parameters of the manufacturing process. Many examples describing the influence of the feeding zone on the efficiency and pressure of the material in the plasticizing system of the press 1,2 can be found in specialist literature. In this respect the most effective proved to be the extruders with a grooved feed section, which allowed one to obtain a tens of percent increase of effectiveness and pressure $3 \div 5$.

Moreover, the quality of the obtained extrudate can be assessed based on the level of mechanical and thermal homogenization of the material, in many cases requiring the use of additional devices, such as static mixers in the process line6. It is especially important in the case of filled materials, among others with nanofillers, which tend to agglomerate, therefore decreasing the quality of the obtained products. According to Głogowska and Sikora7 the modification of polymers by a wide variety of fillers causes numerous changes in processing, mechanical properties and morphology of product structure. Moreover, they confirmed that those properties depend on the proper mixing.

The main construction element of the extruder, significantly influencing the effectiveness of mixing the material and operation of the extruder is, among others, the screw. A proper selection of the geometrical parameters of the screw, both in the feeding (cold) zone and the plasticizing (hot) zone has a direct influence on the pressure and temperature distribution in the transported material. The objective of this work is to present innovative concepts of the screw of an extruder with a plasticizing system arranged to cooperate with an innovative rotational barrel segment. The introduced concept is a fully functional solution, examined in terms of strength and temperature, and therefore suitable for the further stages of 
commercialization.

\section{Concept and geometrical parameters of the screw of the extruder}

The subject of the research was a screw of a extruder, adapted for the rotational segment of the barrel. The geometry of the screw was variable, depending on the work zone. Three concepts of the screw with the working length equal $755 \mathrm{~mm}$ and diameter equal $\varnothing 25 \mathrm{~mm}$ were discussed. The concepts differed in terms of the pitch value and winding depth, which led to different pre-stressing of material, respectively: variant I - 2.09, variant II 2.43 and variant III -2.29 . The construction details of the discussed variants of the screw presented in Fig. 1 also influence the distribution of the reduced stress and the temperature distribution within the screw material.
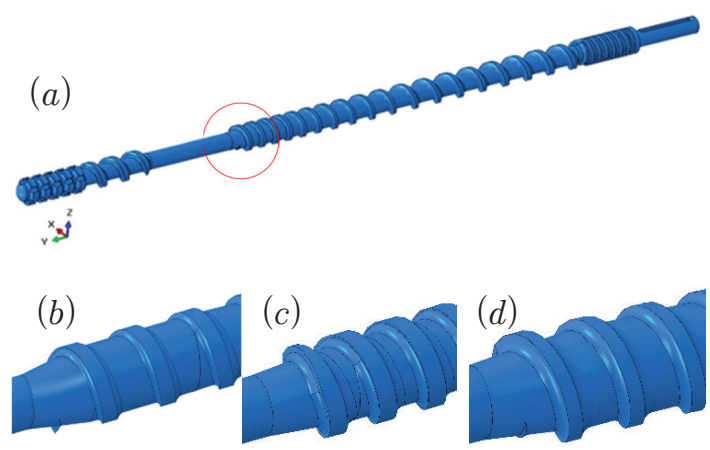

Fig. 1: Geometrical model of the screw: a) general view, b) variant I, c) variant II, d) variant III.

\section{FEM numerical calculations}

\subsection{Methodology of numerical calculations}

The numerical calculations were conducted using the finite element method. Numerical simulations consisted of coupled thermal-displacement analysis, a combination of a static strength analysis and a thermal analysis, allowing one to determine the distributions of stress and temperature in the area of the analysed structure $8 \div 11$. In this case, the general formula for the fully-coupled thermal-stress analysis can be presented as follows:

$$
\left[\begin{array}{c}
K_{u u} K_{u \theta} \\
K_{\theta u} K_{\theta \theta}
\end{array}\right]\left\{\begin{array}{c}
\Delta_{u} \\
\Delta_{\theta}
\end{array}\right\}=\left\{\begin{array}{l}
R_{u} \\
R_{\theta}
\end{array}\right\}
$$

Where: $\Delta v$ and $\Delta \theta$ are the respective corrections to the incremental displacement and temperature, $\mathrm{K}_{\mathrm{ij}}$ are submatrices of the fully coupled Jacobian matrix, and $R_{u}$ and $R_{\theta}$ are the mechanical and thermal residual vectors, respectively. Solving this system of equations requires the use of the asymmetric matrix storage and solution scheme. Furthermore, the mechanical and thermal equations must be solved simultaneously. The method provides quadratic convergence when the solution estimate is within the radius of convergence of the algorithm.

The numerical calculations were a geometrical and physical nonlinear problem, solved using the Newton-Raphson incremental-iterative method $12 \div 15$. The numerical tool applied was a commercial package of the finite element method - the ABAQUS ${ }^{\oplus}$ program.

\subsection{Structure of the discrete model}

The discretization of the geometrical model of the screw was conducted using type C3D4T tetragonal solid elements being 4-node elements with the first-order shape function and full integration, allowing one to consider the thermal degree of freedom in the numerical analysis. The applied method of structure discretization led to creating a discrete model consisting of 127803 finite elements, which resulted in solving a numerical problem with 1294 472 nonlinear equations of the coupled analysis. The general view of the discrete model is presented in fig. 2.

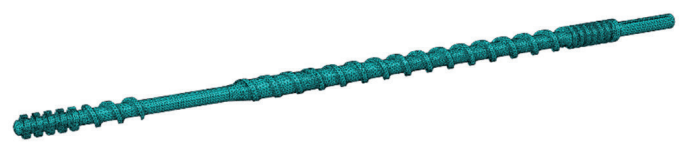

Fig. 2: Discrete model of the screw.

The screw was manufactured from 40HM grade steel, for which an elasto-plastic material model was determined. The material properties are presented in table 1 and 2.

Table 1: Mechanical properties of 40HM grade steel.

\begin{tabular}{|l|l|}
\hline \multicolumn{2}{|l|}{ Mechanical property - steel 40HM } \\
\hline Density $\rho\left[\mathrm{kg} / \mathrm{m}^{3}\right]$ & 7860 \\
\hline Young modulus $\mathrm{E}[\mathrm{Pa}]$ & $2.1 \cdot 10^{5}$ \\
\hline Poisson Coefficient $[-]$ & 0.3 \\
\hline Yield strength $\mathrm{Re}[\mathrm{Pa}]$ & $8.8 \cdot 10^{2}$ \\
\hline Strength limit $\mathrm{Rm}[\mathrm{Pa}]$ & $1.03 \cdot 10^{3}$ \\
\hline Elongation at break [\%] & 10 \\
\hline
\end{tabular}


Table 2: Thermal properties of 40HM grade steel.

\begin{tabular}{|l|l|}
\hline \multicolumn{2}{|l|}{ Thermal property - steel $40 \mathrm{HM}$} \\
\hline Linear expansion coefficient $[1 / \mathrm{K}]$ & $1.2 \cdot 10^{-5}$ \\
\hline Heat conduction coefficient $\lambda[\mathrm{W} / \mathrm{m} \cdot \mathrm{K}]$ & 58 \\
\hline Specific heat $[\mathrm{J} / \mathrm{kg} \cdot \mathrm{K}]$ & 450 \\
\hline
\end{tabular}

\subsection{Boundary conditions and load of the discrete model}

The boundary conditions of the screw model are defined by blocking the translational degrees of freedom of nodes located in the journal bearings in a way presented in fig. 3 .

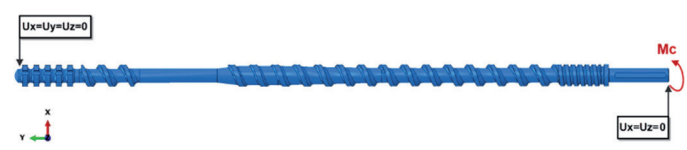

Fig. 3: Definition of the boundary conditions of the screw.

The basic mechanical load was the rotational moment $\mathrm{Mc}=200 \mathrm{Nm}$ applied at the end of the journal (fig. 3). Additional mechanical load was the pressure caused by the transported material, which was imitated by applying pressure to the surface of the screw. The exponential distribution of pressure along the axis of the screw, the maximum value of which was equal pmax $=25 \mathrm{MPa}$ (fig. 4) was defined.

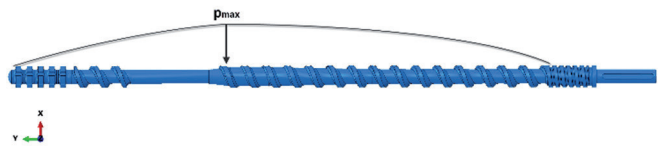

Fig. 4: Exponential distribution of pressure on the surface of the screw.

Thermal load caused by the friction of the transported material in the cold zone of the extruder and the temperature in the hot zone were imitated by applying the temperature fields on the surface of the screw. In order to achieve this the exponential distribution of temperature was defined. Its maximum value occurring in the end of the hot zone was equal $T_{\max }=190^{\circ} \mathrm{C}-$ fig. 5. The initial temperature of the numerical model was assumed to be $T_{0}=22^{\circ} \mathrm{C}$.

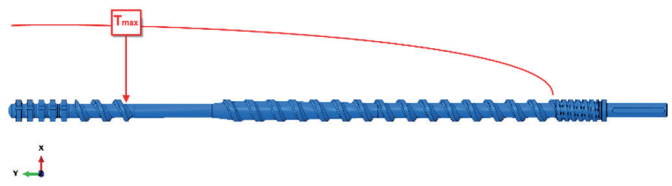

Fig. 5: Exponential distribution of temperature on the surface of the screw

\section{Results of the numerical calculations}

The strength and stiffness analyses of the individual elements of the structure were conducted on the basis of the reduced stress distributions, determined according to the Huber-Mises-Hencky $(\mathrm{H}-\mathrm{M}-\mathrm{H})$ strength hypothesis as well as the maps of node dislocations in the area of the analysed structure. The presented results pertain to the case of mechanical and thermal load of the structure of the screw, equal the constant operation of the device, equal 5 hours. Figure 6 shows the maps of the node dislocations of the screw model expressed in [mm].

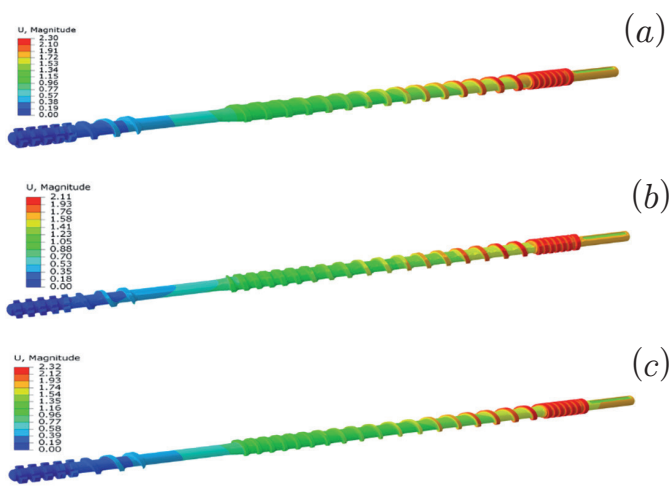

Fig. 6: Maps of the node dislocation of the screw model: a) variant I, b) variant II, c) variant III.

The obtained values of the node dislocation are similar for all three variants and remain in the range $2.11 \div 2.32 \mathrm{~mm}$. Upon referring the obtained results to the dimensions of the screw it can be stated that the structure of the screw remains sufficiently stiff subjected to mechanical and thermal load in the constant operation.

Fig. 7 shows the maps of $\mathrm{H}-\mathrm{MH}$ reduced stress expressed in [MPa], allowing for the assessment of the screw structure strength.

Upon analysing the obtained values of the reduced stress it can be stated that in all the examined variants the level of the maximum stress in similar and oscillates around $\sigma_{z} \approx 875 \div 879 \mathrm{MPa}$. The areas in which the highest gradients of the reduced stress are located are marked in red and occur in the front part (drive application) as well as in the end part of the screw. The obtained values of the reduced stress are high, but do not exceed the value of the yield point. According to the assumed material properties of $40 \mathrm{HM}$ grade steel it is equal $\mathrm{Re}=880 \mathrm{MPa}$. Therefore, the level of the reduced 

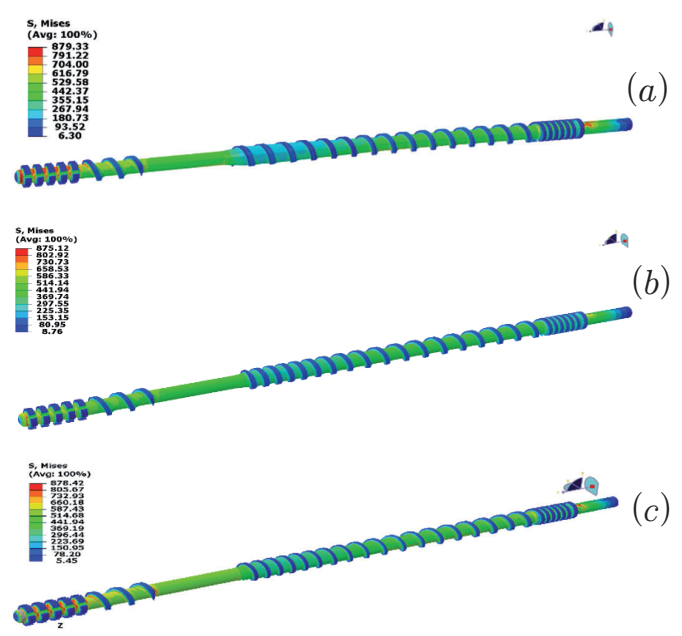

Fig. 7: Maps of the reduced stress $\mathrm{H}-\mathrm{M}-\mathrm{H}$ in the screw structure: a) variant I, b) variant II, c) variant III.

stress occurring in the structure does not threaten the proper work of the structure.

The temperature distributions in the material of the screw were also analysed. Figure 8 shows maps of the temperature expressed in [oC] for the respective construction variants of the screw. The obtained results allow one to determine the temperature distribution in the area of the structure during constant operation of the extruder.

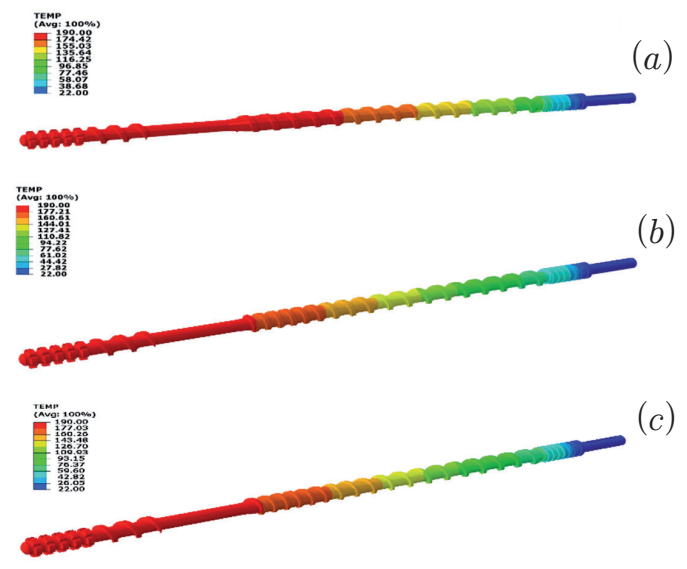

Fig. 8: Maps of temperature distribution in the structure of the screw: a) variant I, b) variant II, c) variant III.

Upon analysing the temperature distributions, it can be observed that in the case of variant I maximum temperature (marked red) occurs on the significant length of the screw, which ensures the optimal granulate plasticizing conditions in the hot zone of the extruder. In the case of variants II and III the area of maximum temperature is significantly shorter, which render those construction solutions less beneficial in the case of the effectiveness of the extruder and optimization of the manufacturing parameters. The direct cause of this phenomenon is the variety of geometrical parameters of the screw windings.

\section{Conclusions}

The study presents innovative construction solutions for the screw of an extruder, adopted for the rotating segment of the barrel. Three construction variants were proposed, differing in the geometrical parameters of the screw windings. A numerical analysis allowed for assessing the strength and temperature distribution in the screw during constant operation of the press. The thermally coupled analysis allowed for considering all aspects of the structure load during the manufacturing process, considering both strength and thermal aspects. The conducted calculations confirmed the sufficient strength and stiffness of the structure subjected to multi-axis load conditions, which ensures the most stable and safe operation of the extruder. An analysis of the temperature distributions during constant operating of the press allowed for selecting the most construction solution - variant I, ensuring the most optimal working conditions, which directly influences the effectiveness of the manufacturing process. The proposed concept of the screw is an innovative solution for the extruder with so-called active grooved feed section and will be commercialized by manufacturing and implementing the extruder to the manufacturing process.

\section{References and Notes}

[1] Sikora J. W., Samujlo B., Stasiek A., Tor-Świątek A. The Mechanical Properties of Plasticized PVC Processed in an Extruder with a Modified Feed Zone. International Polymer Processing 30(3), 359-365 (2015).

[2] Jia, M.-Y, Pa, L., Xue, P., Wang, K.-J., Jin, X.-M. Studies on the Effect of Pellet Size on Positive Conveying in Helically Grooved Single Screw Extruders. Int. Polym. Proc. 28(3), 267273 (2013)

[3] Wortberg J. Screw And Barrel Design For Grooved Feed Vs. Smooth Bore Extruders, SPE-ANTEC, San Francisco, 1-5 (2002).

[4] Sasimowski E., Sikora J., Królikowski B. Effectiveness of poly- 
ethylene extrusion in a single-screw grooved feed extruder. Polimery 59(6), 505-510 (2014).

[5] Sikora J.W. The effect of construction modifications of the extruder barrel grooved zone on the autothermal extrusion process. Polimery 43(9), 548-554, (1998).

[6] Bruce A. Davis, Paul J. Gramann, Maria Del P. Noriega E., Tim A. Osswald Grooved Feed Single Screw Extruders - Improving Productivity and Reducing Viscous Heating Effects.

[7] Głogowska K., Sikora J., Blase J. The use of untreated neuburg silliceous earth as filler for high-density polyethylene. Tehnicki Vjesnik-Technical Gazette 25(6), 1581-1586 (2018).

[8] Abaqus HTML Documentation (2018).

[9] Yang Z.J., Harkin-Jones E., Menary G.H., Armstrong C.G. Coupled temperature-displacement modelling of injection stretch-blow moulding of PET bottles using Buckley model. Journal of Materials Processing Technology 153-154, 20-27 (2004).

[10] Senguptaa J., Cockcroftb S.L., Maijerb D.M., Larouchec A. Quantification of temperature, stress, and strain fields during the start-up phase of direct chill casting process by using a 3D fully coupled thermal and stress model for AA5182 ingots. Materials Science and Engineering: A 397(1-2), 157177 Apr. (2005).

[11] Kulkarni S. M., Rubin C. A. and Hahn G. T. Elasto-Plastic Coupled Temperature-Displacement Finite Element Analysis of Two-Dimensional Rolling-Sliding Contact With a Translating Heat Source. ASME J. Tribol 113(1), 93-101 (1991).

[12] Bakari H.R, Adegoke T.M, and Yahya A.M Application of Newton-Raphson method to non-linear models. International Journal of Mathematics and Statistics Studies 4(4), 21-31 (2016).

[13] Horiguchi S. The Formulas to Compare the Convergences of Newton's Method and the Extended Newton's Method (Tsuchikura-Horiguchi Method) and the Numerical Calculations. Applied Mathematics 7 (1), 40-60 (2016).

[14] Janicke L. Kost A. Convergence properties of the NewtonRaphson method for nonlinear problems. IEEE Transactions on Magnetics 34(5), 2505 - 2508 (1998).

[15] Verbeke J., Cools R. The Newton-Raphson method. International Journal of Mathematical Education in Science and Technology 26(2), 177-193 (1995).

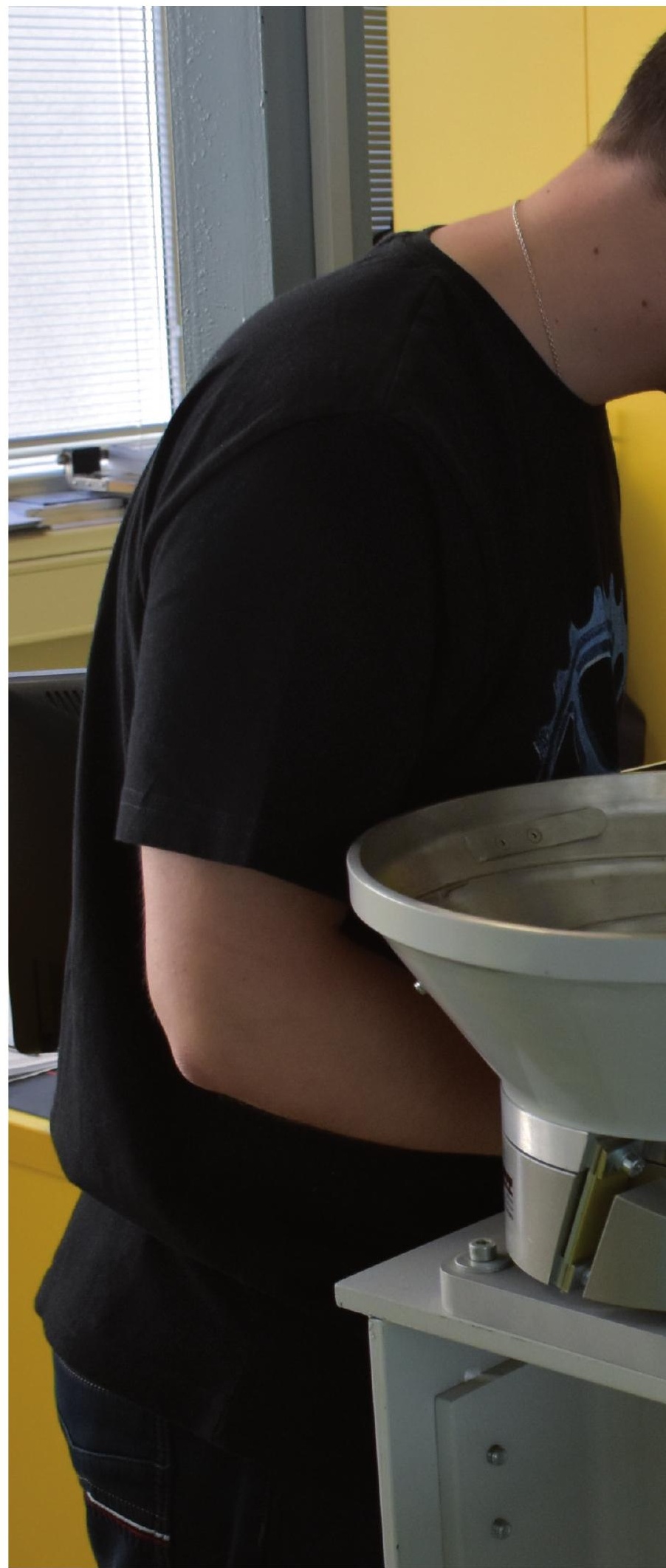

